\title{
Accurate vessel width measurement from fundus photographs: a new concept
}

Salwan M B Rassam, Vinod Patel, Olaf Brinchmann-Hansen, Oddbjørn Engvold, Eva M Kohner

\begin{abstract}
Accurate determination of retinal vessel width measurement is important in the study of the haemodynamic changes that accompany various physiological and pathological states. Currently the width at the half height of the transmittance and densitometry profiles are used as a measure of retinal vessel width. A consistent phenomenon of two 'kick points' on the slopes of the transmittance and densitometry profiles near the base, has been observed. In this study, mathematical models have been formulated to describe the characteristic curves of the transmittance and the densitometry profiles. They demonstrate the kick points being coincident with the edges of the blood column. The horizontal distance across the kick points would therefore indicate the actual blood column width. To evaluate this

these techniques is dependent on the characteristic photosensitivity calibration curves of the photographic film used. This is potentially another source of error.

At the Hammersmith hospital, a computer assisted digital image analyser is used to measure the light transmittance through film negatives of monochromatic fundus photographs, ${ }^{90}$ and displays them on a screen as transmittance profiles. Two distinct skew points termed 'kick points' have been observed on the slopes in the transmittance profiles near the base of the curves (Fig 1). These kick points are also seen in densitometry profiles. ${ }^{811}$ We set out to determine the source of these kick points, and to study the relation between the blood column and the vessel wall using mathematical models of hypothetical artery and vein. These models were tested using plastic tubing and human retinal photographs.
\end{abstract} hypothesis, blood was infused through two lengths of plastic tubing of known diameters, and photographed. In comparison with the known diameters, the half height underestimated the blood column width by $\mathbf{7 \cdot 3 3 \%}$ and $6.46 \%$, while the kick point method slightly overestimated it by $1.40 \%$ and $0.34 \%$. These techniques were applied to monochromatic fundus photographs. In comparison with the kick point method, the half height underestimated the blood column width in veins by $16.67 \%$ and in arteries by $15.86 \%$. The characteristics of the kick points and their practicality have been discussed. The kick point method may provide the most accurate measurement of vessel width possible from these profiles.

(Br f Ophthalmol 1994; 78: 24-29)

Diabetic Retinopathy Unit, Department of Medicine, Royal Postgraduate Medical School, Hammersmith

Hospital, London

S M B Rassam

V Patel

E M Kohner

Department of Ophthalmology, University Hospital of Trondheim, N-7006

Trondheim, Norway

O Brinchmann-Hansen

Institute of Theoretical Astrophysics, University of Oslo, PO Box 1029 Blindern N-0315, Oslo 3, Norway

O Engvold

Correspondence to:

Professor Eva M Kohner, Diabetic Retinopathy Unit, Department of Medicine, Royal Postgraduate Medical School, Hammersmith Hospital, Du Cane Road, London W12 0NN.

Accepted for publication 17 August 1993

\section{Materials and method}

MATHEMATICAL MODEL

The mathematical model of the hypothetical
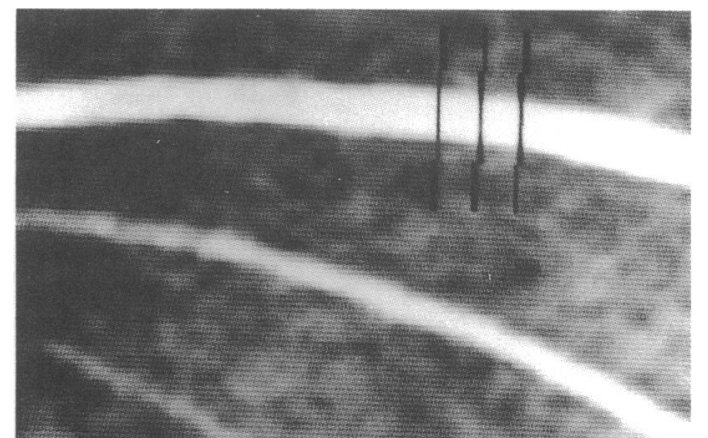

The retinal vessels are the only part of the central circulation that can be viewed directly and studied in detail. Retinal vessel width changes have been utilised in the study of various physiological and pathological conditions. ${ }^{123} \mathrm{With}$ the development of new techniques that accurately measure the velocity of red cells such as bidirectional laser Doppler velocimetry ${ }^{4}$ and scanning laser ophthalmoscopy, ${ }^{5}$ precise measurements of retinal vessel width become important for the determination of retinal volume blood flow. At present, the most commonly used techniques in determining vessel width from retinal photographs are microdensitometry which measures the density profile, film scanners that record photographic image transmittance, and film densities to provide film exposure profiles. ${ }^{67}$ The only available method to derive an estimate of the vessel width from these profiles, uses the arbitrary assumption of the width at the half height of the profiles as a measure of the blood column width (Fig 1). ${ }^{8}$ The width at the half height using
Figure 1 Monochromatic fundus photograph, displaying the transmittance profile of a vessel from the photographic negative. The half height, the kick points, and the light reflex are shown. 
Figure 2 A cross sectional model of a vessel. The inner circle represents the blood column. The outer circle represents the vessel wall, $(x)$ is derived from the radius of the blood column and the thickness of the vessel wall: $(z)$ is the position from the vessel edge at increments of $1 \mu \mathrm{m} ;(y)$ is the height to be determined using Pythagoras's theorem.

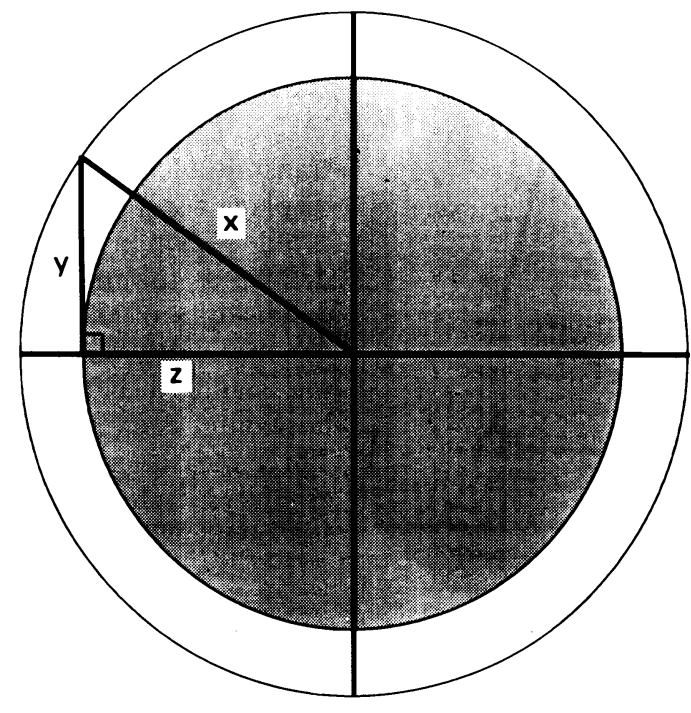

blood vessel was depicted by two concentric circles, the inner circle representing the blood column and the outer circle representing the vessel wall (Fig 2). In vivo, the thickness of an arterial wall is approximately one sixth that of the blood column width, ${ }^{12}$ and the venous wall one tenth the width of its blood column. ${ }^{13}$ Therefore, we proposed two models; an arterial model with a blood column width of $100 \mu \mathrm{m}$ and a vessel wall thickness of $15 \mu \mathrm{m}$, and a venous model with the same blood column width of $100 \mu \mathrm{m}$ but the wall thickness was made $10 \mu \mathrm{m}$. The assumptions (i) that the vessel is round and (ii) the vessel image was taken perpendicular to the photographic film, were made.

Light from the fundus camera is reflected back by various structures in the retina and choroid. As the blood vessels absorb some of the light reflected from the retinal/choroidal background, the relative darkness of a vessel may be expressed in terms of its opacity (assuming single transmission through the vessels). It may be shown that the transmitted intensity of the blood vessel, relative to the unobscured retinal background intensity, may be expressed by Lambert's law for absorption. ${ }^{14}$ Lambert's law states that if $\mathrm{I}_{\mathrm{o}}$ is the original intensity, $I$ is the intensity after passing through a thickness of 1 of a material whose absorption coefficient is $\alpha$ :

$$
I=I_{0} \cdot e^{-\alpha l}
$$

Since Then

Transmittance $(T)=I / I_{o}$

$$
\mathrm{T}=\mathrm{e}^{-\alpha \mathbf{l}}
$$

To calculate transmittance through the blood and vessel wall, the absorption coefficients for the $\operatorname{blood}\left(\alpha_{c}\right)$ and vessel wall $\left(\alpha_{w}\right)$, and the light path length (l) need to be determined.

As light passes through a blood vessel, some of the light is absorbed by the wall and the blood. The fraction of light absorbed by the artery wall (absorbance) at a wavelength of $570 \mathrm{~nm}$ is 0.145 and for the venous wall 0.149 as calculated from the work of Flower et al. ${ }^{15}$ From these absorbance data, the absorption coefficients were calculated using the formula:

$$
A=I_{0}\left(1-e^{-a l}\right)
$$

where $A=$ absorbance

$\mathrm{e}=2 \cdot 71828$ is the base of the natural logarithm

$\alpha=$ absorption coefficient of the medium

$\mathrm{l}=$ length of the light path through the medium

$I_{0}=$ normal background intensity

The absorption coefficient of the arterial wall was calculated to be $104.7 \mathrm{~cm}^{-1}$, and for the venous wall $161 \cdot 1 \mathrm{~cm}^{-1}$. The millimolar absorption coefficient of an undiluted, haemolysed sample of blood, at $570 \mathrm{~nm}$, is $11 \cdot 27$ l. $\mathrm{mmol}^{-1} \cdot \mathrm{cm}^{-1}$, in accordance with van Assendelft's data. ${ }^{16}$ The normal range of haematocrit between the 5 th and 95 th percentile for males aged 3 to 74 years, in all races, is 0.356 to $0 \cdot 482 .{ }^{17}$ Within this range, a haematocrit of 0.467 equates to a haemoglobin concentration of $10 \mathrm{mmol} . \mathrm{l}^{-1}$. Correcting the absorption coefficient of blood to this haemoglobin concentration, the value would be $112.7 \mathrm{~cm}^{-1}$. This value is true at the logarithmic base of 10; however, in our calculations of the absorption coefficients of the vessel walls, we used the natural logarithm. The natural $\log$ value of the blood absorption coefficient would therefore be $(112 \cdot 7 \times 2 \cdot 3026) 259 \cdot 5$ $\mathrm{cm}^{-1}$.

The light's path length through the vessel was determined from the proposed model using Pythagoras's theorem. The respective lengths through the blood colum $\left(l_{c}\right)$ and the vessel wall $\left(l_{w}\right)$ were calculated as a function of the distance from the central line starting from the edge of the vessel at increments of $1 \mu \mathrm{m}$ (Fig 2). From the absorption coefficients and the light path lengths, the absorption of light $(\alpha \mathrm{l})$ was calculated for the vessel wall $\left(\alpha_{w} l_{w}\right)$ and the blood column $\left(\alpha_{c} l_{c}\right)$ separately. As the absorption coefficient of the vessel wall is different from that of the blood column, the overall absorption ( $\alpha \mathrm{l})$ for the blood vessel as a whole was calculated using the formula:

$$
\alpha \mathrm{l}=\alpha_{\mathrm{w}} \mathrm{l}_{\mathrm{w}}+\alpha_{\mathrm{c}} \mathrm{l}_{\mathrm{c}}
$$

where $\alpha_{\mathrm{w}}=$ absorption coefficient of the vessel wall

$1_{w}=$ light path length through the thickness of the vessel wall

$\alpha_{c}=$ absorption coefficient of the blood column

$l_{c}=$ light path length through the thickness of the blood column

Transmittance values for the vessel wall and blood column separately and both together were obtained. A graph of the transmittance on the $y$ axis versus the distance across the vessel on the $\mathrm{x}$ axis was plotted. The blood column curves (Figs $3 \mathrm{~A}, 4 \mathrm{~A}$ ) and the vessel wall curves (Figs 3B, 4B) were obtained. When the overall transmittance curves for the artery and the vein were determined (Figs 3C, 4C), they corresponded to the transmittance profiles of retinal vessels as determined by the photographic scanners and image analysis systems from photographic images (Fig 1), without the light reflex from the centre of the vessel as this was not calculated in the mathematical models. It clearly shows the kick points on either side of the profiles, as well defined skew points. These points mark the edges of the blood 


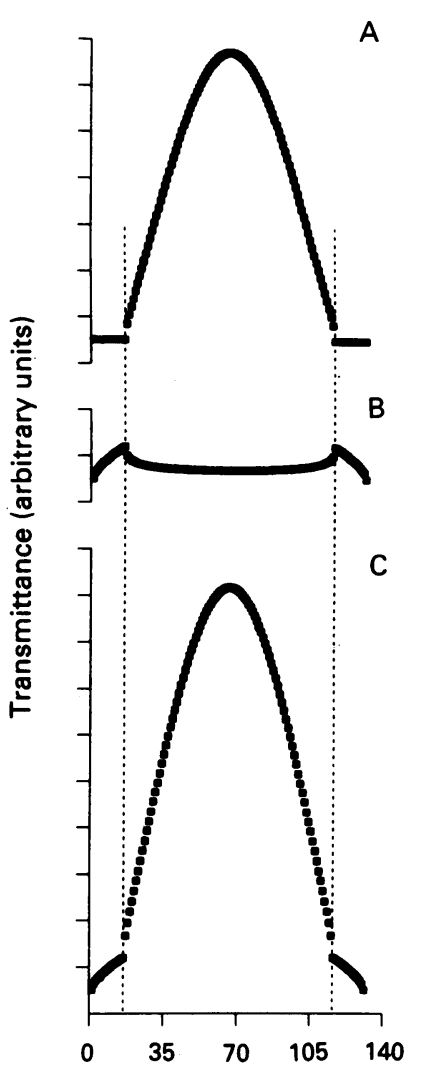

Distance from vessel edge $(\mu \mathrm{m})$

Figure 3 Transmittance profiles of the arterial model. (A) Transmittance of the blood column. (B) Transmittance of the arterial wall. $(C)$ The overall transmittance of the artery, showing the kick points on either side of the slopes. The kick points correspond to the highest transmittance of the arterial wall (the longest path length of light) and the start of the blood column transmittance.
Distance from vessel edge $(\mu \mathrm{m})$

Figure 4 Transmittance profiles of the venous model. (A) Transmittance of the blood column. (B) Transmittance of the venous wall. $(C)$ The overall transmittance of the vein, showing the kick points on either side of the slopes. The kick points correspond to the highest transmittance of the venous wall (the longest path length of light) and the start of the blood column transmittance.

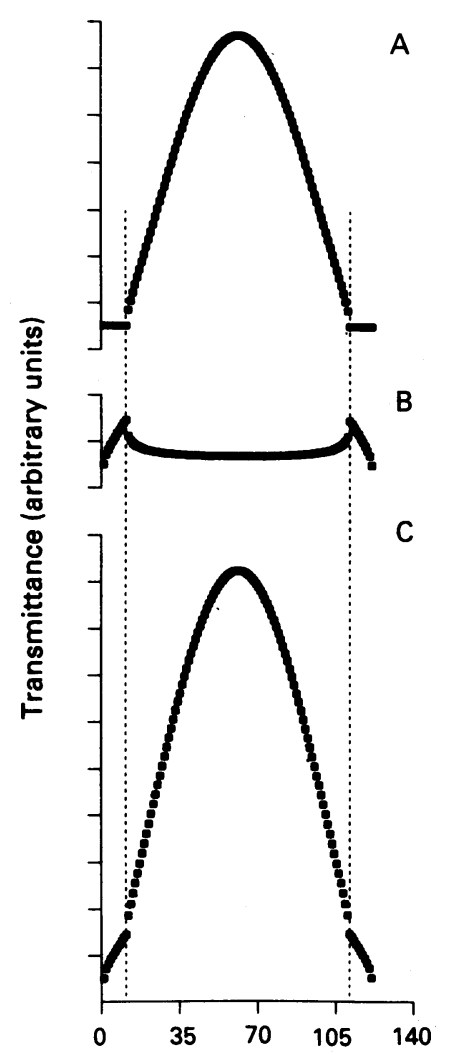

the film the photographic negatives were projected through a video camera into a Context Vision GOP-302 digital image analyser (Context Vision, Linköping, Sweden) which measures the transmittance of the photographic negative and displays it in a profile similar to the retinal vessel profile shown in Figure $1 .{ }^{10}$ The 'kick points' are defined as the first distinct skew points on the downslopes of the profiles (Fig 5). The blood column width was then determined using both the standard half height technique and the horizontal distance across the two kick points. Three measurements on each of the two photographs on each tube were taken, by one observer (a total of six measurements for each tube).

\section{IN VIVO MEASUREMENT}

From these models we proceeded to test the kick point method on photographs of human fundi. Analysis of fundus photographs of all 10 healthy volunteers from an ongoing clinical study was undertaken. The age range was 28 to 38 years (mean (SD), $31 \cdot 9(3 \cdot 2)$ ). Informed consent was obtained, and pupillary dilatation was achieved with $0.5 \%$ tropicamide eye drops. Fundus photographs were taken using the same standard Zeiss $30^{\circ}$ camera, with the $570 \mathrm{~nm}$ green filter. The camera was linked to an electrocardiograph through a time delay processor. This time delay processor triggers the shutter of the camera at the same predetermined point of the cardiac cycle, thus eliminating the effect of pulsatility on vessel widths during the cardiac cycle. In our labora-

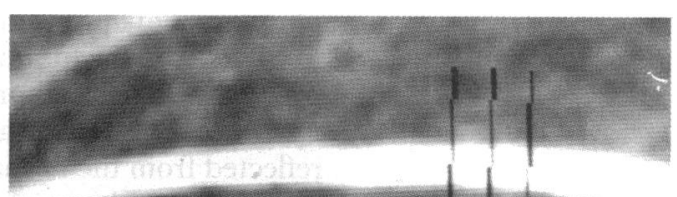

column. Therefore, by taking the horizonta distance from one kick point to the other, an accurate measurement of the width of the blood column can be obtained (Fig 5).

EXPERIMENTAL MODEL

To determine whether the mathematical models were accurate in determining the real width of the blood column across the kick points in the transmittance profiles, an experimental model was designed using plastic tubing of two different calibres. The tubes were placed on a graticule to account for the effect of magnification during the measurements. They were perfused with whole human blood and EDTA added to prevent clotting. This was to simulate a blood vessel. The experiment was done in two stages. In the first stage, the tubes and graticule were placed on a microscope stage. Using the microscope it was possible to measure accurately the blood column width. Three readings of each tube were taken by two independent observers. The second stage was to photograph the same point on the perfused tubes using a simulated background similar to the retinal background, with a standard Zeiss $30^{\circ}$ fundus camera (Carl Zeiss, Oberkochen, Germany) and a $570 \mathrm{~nm}$ green filter.' A Kodak Technical Pan film (Kodak, Rochester, NY, USA) was used. Two photographs of each tube were taken. After developing

Figure 5 Monochromatic fundus photograph, displaying the transmittance profile of a vessel. The two kick points have been marked. The distance between these kick points represents the true width of the blood column. 
tory, the photographs are taken in end diastole. The $570 \mathrm{~nm}$ green filter enhances the blood contrast in the retinal vessels. The blood vessels would therefore appear light with respect to the fundus background in the resultant negatives (Figs 1, 5). The photographic negatives were then analysed using the Context Vision digital image analyser as described above. On each subject three fundus photographs were analysed. Three measurements of blood column width were determined on each of the three photographs from each subject, using both the half height and the kick point methods. The measurements were conducted on the superior temporal artery and the corresponding vein, within half a disc diameter from the disc edge. The magnification of the vessels by the Zeiss fundus camera was corrected for the ocular refraction of each subject in accordance with the tables of Bengtsson and $\mathrm{Krakau}^{18}$ Therefore retinal vessel widths were expressed in micrometres $(\mu \mathrm{m})$ to a high degree of precision.

\section{Statistical analysis}

Data from the experimental model were analysed using a paired $t$ test. The difference of the kick point and the half height methods in comparison with the microscopic measurement is expressed in terms of percentage error. The differences between measurements of retinal vessels using the two methods are expressed as a percentage of the kick point width. Student's $t$ test was employed to define statistical differences between the groups. Confidence intervals (95\% $\mathrm{CI}$ and $99 \% \mathrm{CI}$ ) were determined for the differences between the two methods of measurements, as described by Bland and Altman. ${ }^{19}$

\section{Results}

The results from the experimental model are shown in Table 1. The half height method significantly underestimated the blood column width by $7.33 \%$ in tube $1(\mathrm{p}<0.001)$ and $6.46 \%$ in tube 2 ( $p>0.001)$. A degree of underestimation was predicted from the theoretical model

Table 1 Results from the experimental model (mean (SD))

\begin{tabular}{lcll}
\hline Method & Tube 1 $(\mu \mathrm{m})$ & Tube 2 $(\mu \mathrm{m})$ & $\begin{array}{l}\text { No of } \\
\text { photos }\end{array}$ \\
\hline Microscope & $987 \cdot 50(8.96)$ & $735 \cdot 00(4 \cdot 91)$ & 2 \\
Half height & $915 \cdot 15(5.97)^{\star}$ & $687 \cdot 50(7 \cdot 45)^{\star}$ & 2 \\
Kick point & $1001 \cdot 30(6.89)$ & $732 \cdot 50(3.64)$ & 2 \\
Percentage error: & $-7 \cdot 33 \%$ & $-6.46 \%$ & \\
Half height & $+1.40 \%$ & $+0.34 \%$ & \\
Kick point & & & \\
\hline
\end{tabular}

${ }^{\star} \mathrm{p}<0.001$ in comparison with the microscopic measurement.

Table 2 Results of measurements from retinal photographs (mean (SD))

\begin{tabular}{lccc}
\hline Method & Vein $(\mu \mathrm{m})$ & Artery $(\mu \mathrm{m})$ & $\begin{array}{c}\text { No of } \\
\text { photos }\end{array}$ \\
\hline Half height & $141.49(19.42)$ & $112.07(16.94)$ & 30 \\
Kick point & $170.04(23.24)$ & $132.97(17.46)$ & 29 \\
Difference & $28.55(9 \cdot 91)$ & $20.90(4 \cdot 73)$ & \\
Percentage difference & $(\mathrm{p}<0 \cdot 001)$ & $(\mathrm{p}<0 \cdot 001)$ & \\
$95 \%$ CI & $16.79 \%(5 \cdot 83 \%)$ & $15 \cdot 72 \%(3 \cdot 56 \%)$ & \\
$99 \%$ CI & $+12.92 \%$ to $+20 \cdot 42 \%$ & $+13.15 \%$ to $+18 \cdot 57 \%$ & \\
\hline
\end{tabular}

Number of subjects $=10$.

$\mathrm{CI}=$ confidence interval.
(Fig 1). The kick point method slightly but not significantly overestimated by $1.40 \%$ in tube 1 $(p=0.312)$ and $0.34 \%$ in tube $2(p=0.939)$.

The results from the retinal photographs are shown in Table 2. From the total of 30 photographs analysed, only one photograph did not show the kick points on either the artery or vein; this was because of poor focusing. However, the other two photographs of the same subject did show the kick points on the artery and vein. It was possible to use the half height method on all photographs for arteries and veins.

The variability of the half height and kick point methods were determined from the coefficient of variation $(S D \times 100 /$ mean $)$ of the three individual measurements on each photograph. This was to show the reproducibility of the two techniques. The coefficients of variation were averaged for each method and for arteries and veins and the standard deviation then determined. For veins, the variability of the half height method was $2 \cdot 81 \%$ (SD $2 \cdot 17 \%$ ), and that of the kick point method was slightly better at $2.45 \%$ (SD $1.85 \%$ ). In arteries the variability of the kick point method was also better $(2.98 \%$ (SD $2 \cdot 11 \%)$ ) than with the half height method (3.94\% (SD 2.16\%)).

The range of the venous widths using the half height method was 113.68 to $176.99 \mu \mathrm{m}$, and the kick point method was $145 \cdot 16$ to $211 \cdot 16 \mu \mathrm{m}$. The $95 \%$ confidence interval for the difference between the two methods for veins was 21.47 to $35.63 \mu \mathrm{m}$. The $99 \%$ confidence interval for the same was 18.36 to $38.74 \mu \mathrm{m}$. The range of the arterial widths using the half height method was 83.70 to $136 \cdot 10 \mu \mathrm{m}$, and the kick point method was $107 \cdot 45$ to $161 \cdot 28 \mu \mathrm{m}$. The $95 \%$ confidence interval for the difference between the two methods for arteries was 15.38 to $26.42 \mu \mathrm{m}$. The $99 \%$ confidence interval for the same was 12.96 to $28 \cdot 84 \mu \mathrm{m}$.

\section{Discussion}

This study provides a mathematical model that explains the characteristics of the transmittance and densitometry profiles across a blood vessel, using available data on the physical properties of blood and vessel wall. It shows that the kick points occur where the wall meets the most lateral extent of the blood column, providing a rationale for using the horizontal distance across the kick points as the blood column width.

Although the use of an automated, computer assisted digital image analysis system significantly reduces the inter and intraobserver variability of retinal vessel width measurements, in comparison with observer driven methods, ${ }^{10}$ the slight but non-significant $(1.4 \%$ and $0.34 \%)$ overestimation of tube width using the kick point method seen in the experimental model may be due to observer and inherent instrumentation error.

The kick points are dependent on two facts: (a) the light path through the vessel wall is greatest at the lateral interface between the blood column and the vessel wall (Figs 3, 4); (b) the absorption coefficient is different for the blood from that of the vessel wall. If the blood and vessel wall had the same optical densities $\left(\alpha_{c}=\alpha_{w}\right)$ 

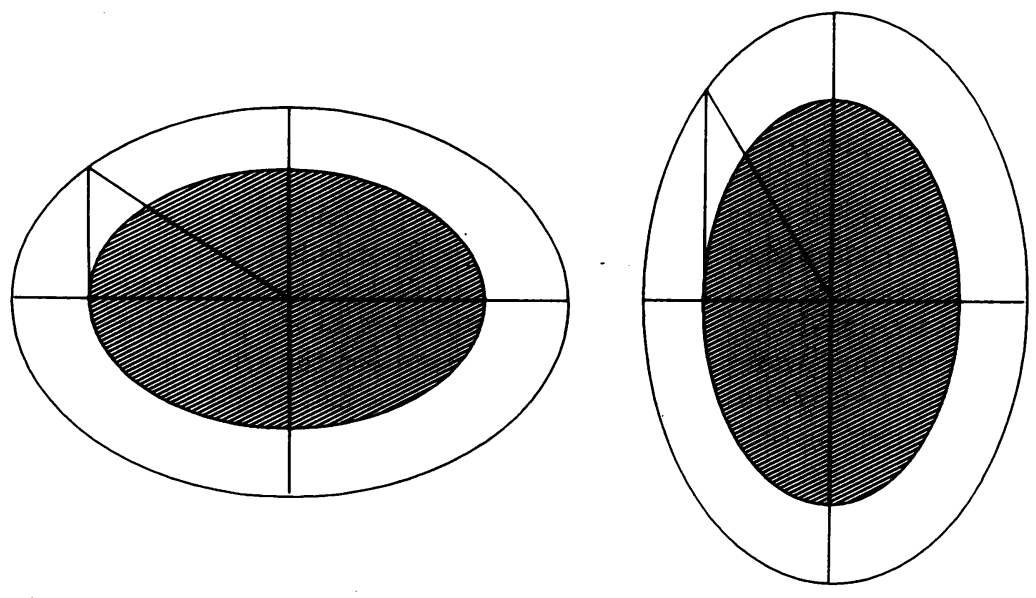

Figure 6 Cross section through elliptical vessels with the widest diameter oriented horizontally and vertically. The longest light path length through the vessel wall still remains at the lateral interface between the blood column and the vessel wall.

then the kick points will not appear. Even if the vessels have an elliptical cross section, whether horizontal or vertical (Fig 6), the light path through the vessel wall would still be greatest at the lateral interface between the blood column and vessel wall, and therefore the kick points would still appear in the profiles. The width of elliptical vessels will vary, depending on whether they are horizontally or vertically oriented, in whichever method is used.

The assumption of single transmission of light through the vessel (and not double) does not matter as long as the vessels are optically thin that is, $\alpha l \leqslant 1$. From the absolute data available for $\alpha_{c}$ and $\alpha_{w}$, it would seem that the vessel walls are optically thin. Even if they were optically thick, the profile shape will be affected somewhat, but the position of the kick points would remain unchanged.

The plastic tubing used was larger than true retinal vessels. However, this did not affect the results since the tubing was resolved in the same fundus camera as the retinal photographs, the relative position and strength of the kick points do not change by scaling to larger diameters. The central part of the profile may change, but only if the optical thickness $(\alpha)$ of the tubing is $>1$ which, anyhow, is irrelevant to the position of the kick point.

The optimal intensity level for the measurement of intensity and, therefore, transmittance profiles from the photographic images was used. The half height method is influenced by the response function of the film emulsion on the recorded image; as image intensities outside the dynamic range of the film used will have a different response to the light reflected by the retinal vessels and the retinal background, producing a variation in the height of the transmittance curves. ${ }^{7}$ This is not so for the kick point method as it is dependent on a particular characteristic of the profile, namely the kick points. Therefore the position of the kick points is not reliant on the photosensitivity calibration of the photographic film, contrary to all other methods. The kick point method thus avoids the otherwise most severe of the error sources associated with the use of the photographic film. Sources of error that may affect the reproducibility both methods would be the accuracy in finding the same point across the vessel in different fundus photographs, and probably the variation of vasomotion and pulsatility. In this study, the effect of pulsatility was eliminated by the use of an electrocardiograph linked to a delay processor allowing the camera to obtain the photographs at the same predetermined point of the cardiac cycle. The reproducibility of the technique was assessed as for the plastic tubing. The variability of the kick point method was less than the half height method in both veins $(2.45 \%$ (SD $1 \cdot 85 \%$ ) vs $2 \cdot 81 \%$ (SD $2 \cdot 17 \%$ )) and arteries $(2 \cdot 98 \%$ (SD $2 \cdot 11 \%$ ) vs $3 \cdot 94 \%$ (SD $2 \cdot 16 \%)$ ).

To observe the kick points does require photographs with good focusing, as the shape of the slope of the intensity curve at the edge of the blood column changes with focusing. Although this has no effect on the position and the degree of separation of the kick points, this would make the detection of the kick points difficult. In contrast the half height method is not significantly affected by focusing errors since it does not require the detection of particular points of the intensity curves. ${ }^{20}$ For this reason, the half height method may be more practical, since it can be determined from most photographs. In our study, however, 29 out of 30 photographs showed the kick points $(96.67 \%)$ for both arteries and veins. Only one photograph of one subject was poorly focused; the other two photographs of the same subject showed the kick points clearly. Therefore, the kick point method could be used in any study limited only by focusing errors.

In this paper, the emphasis was made on blood column width rather than whole vessel width, as the blood column is more important when calculating the blood volume flow rate, in the retinal vessels. In comparison to the kick point method of blood column measurement, the half height method underestimated internal venous widths by $16.67 \%$ and internal arterial widths by $15 \cdot 86 \%$ (assuming the kick point to be correct). Calculating the volumetric flow rate in the retinal vessels from vessel diameter involves squaring of the diameter values. ${ }^{13}$ Such an underestimation would produce markedly lower values for volume flow. The $95 \%$ confidence intervals for the percentage error in determination of the vessel width (Table 2), suggest that the potential error in calculation of retinal volume flow would be $27 \cdot 58 \%$ to $45 \cdot 01 \%$ in veins and $28.03 \%$ to $40.59 \%$ in arteries.

It is also theoretically possible, from the mathematical model, to measure the vessel wall thickness from the transmittance and densitometry profiles, by measuring the horizontal distance between a kick point and the end of the lower curve, connecting to the background retinal structures. In practice, this was not possible due to the variation in the lateral ends of the lower curves corresponding to the dark zone seen adjacent to the vessel and variation in background intensity due to structural variation such as pigmentation and nerve fibre layer density. There will also be an additional effect of the vessel lateral shadows on the background intensity. These factors, however, will not interfere with the appearance of the kick points as they are usually situated away from the lateral ends of the blood column corresponding to the kick points.

A factor that will influence the profile and the 
position of the kick points of the mathematical model is a potential variation of the coefficient $\alpha_{c}$ along the sight line through a blood column. This would be the case if, for example, the density of red cells increases towards the centre of the blood column. If so, once a variation in $\alpha_{c}$ through a blood column is known, it can be accounted for, in profiles derived from a numerical model like the one discussed in this paper. Therefore, a simple model like the one used here will not reproduce the finer details of the profiles of retinal vessels, but would still be a valid demonstration of the usefulness of the kick points.

The contribution of the plasma zone on the transmittance profiles has not been calculated. It may contribute to the vessel wall curve as it lacks red cells and that makes it less dense. However, its thickness is so small compared with the rest of the vessel wall (approximately 1\%), its effect would be negligible. ${ }^{21}$

The kick points have also been observed in microdensitometry profiles of both retinal photographs ${ }^{6711}$ and scanning laser ophthalmoscopy images.

This paper provides the theoretical basis for questioning the use of half height in measuring the vessel widths from transmittance and densitometry profiles. The essential parameters in any haemodynamic study in health and disease are blood velocity and vessel diameter. The kick point method may provide the most accurate measurement of vessel width possible from these profiles.

We would like to express our gratitude to Dr van Assendelft for his helpful suggestions and discussions.

1 Brinchmann-Hansen O, Myhre K, Sandvik L. The light reflex in retinal vessels and its relation to age and systemic blood pressure. Acta Ophthalmol (Kbh) 1987; 65 (Suppl): 206-12.

2 Lanigan LP, Clark CV, Allawi J, Hill DW, Keen $H$ Responses of the retina circulation to systemic autonomic stimulation in diabetes mellitus. Eye 1989; 3: 39-47.
3 Grunwald JE, Riva CE, Brucker AJ, Sinclair SH, Petrig BL. Altered retinal vascular responses to $100 \%$ oxygen breathing

4 Riva CE, Feke GT, Eberli B, Benary V. Bidirectional LDV system for absolute measurement of blood speed in retinal vessels. Applied Optics 1979; 18: 2301-6.

5 Bertram B, Wolf S, Fiehöfer S, Schulte K, Arend O, Reim M. Retinal circulation times in diabetes mellitus type I. Br f Ophthalmol 1991; 75: 462-5.

6 Delori FC, Fitch KA, Feke GT, Deupree DM, Weiter JJ. Evaluation of micrometric and microdensitometric methods for measuring the width of retinal vessel images on fundus photographs. Graefes Arch Clin Exp Ophthalmol 1988; 226: 393-9.

7 Brinchmann-Hansen O, Engvold O. Microphotometry of the blood column and the light streak on retinal vessels in fundus photographs. Acta Ophthalmol (Kbh) 1986; 179 (Suppl): 9-19.

8 Brinchmann-Hansen $\mathrm{O}$, Heier H, Myhre K. Fundus photography of width and intensity profiles of the blood column and light reflex in retinal vessels. Acta Ophthalmol (Kbh) 1986; 179 (Suppl): 20-8.

9 Delori FC, Gragoudas ES, Francisco R, Pruett RC. Monochromatic ophthalmoscopy and fundus photography: the normal fundus. Arch Ophthalmol 1977; 95: 861-8.

10 Newsom RSB, Sullivan PM, Rassam SMB, Jagoe R, Kohner EM. Retinal vessel measurement: comparison between observer and computer driven methods. Graefes Arch Clin observer and computer driven meth

11 Eaton AM, Hatchell DL. Measurement of retinal vessel width using computerised image analysis. Invest Ophthalmol Vis Sci 1988; 29: 1258-64.

12 Wise GN, Dollery CT, Henkind P. Structure of retinal vessels. In: The retinal circulation. New York: Harper and Row, 1971; chapter 3: 34-54.

13 Ganong WF. Dynamics of blood and lymph flow. In: Review of medical physiology. 12th Ed. California: Lange, 1985; chapter 30: 470-84.

14 Weast RC, Miscellaneous. In: Weast RC, Astle MJ, Beyer WH, eds. CRC handbook of chemistry and physics. Florida: CRC Press, 1986: F65.

15 Flower RW, McLeod DS, Pitts SM. The effect of blood on ocular fundus reflectance and determination of some optical properties of retinal blood vessels. Invest Ophthalmol Vis $S c i$ 1978; 17: 562-5

16 Van Assendelft OW. Spectrophotometry of haemoglobin derivatives. Assen, The Netherlands: van Gorum, 1970: 55-7.

17 Fulwood R. Haematological and nutritional biochemistry data for persons 6 months to 74 years of age: United States, 197680. Vital and health statistics. DHHS Publication No (PHS) 1981; 11: 83-1682.

18 Bengtsson B, Krakau CET. Some essential optical features of the Zeiss fundus camera. Acta Ophthalmol $(K b h) 1977 ; 55$ : 123-31.

19 Bland JM, Altman DG. Statistical methods for assessing agreement between two methods of clinical measurement. Lancet 1986; i: 307-10.

20 Heier H, Brinchmann-Hansen O. Reliable measurements from fundus photographs in the presence of focusing errors. Invest Ophthalmol Vis Sci 1989; 30: 674-7.

21 Bulpitt CJ, Dollery CT, Kohner E. The marginal plasma zone in the retinal microcirculation. Cardiovasc Res 1970; 4; 207-12. 